\title{
Chemotherapy-induced Neutropenic Septic Shock in Gynecologic Cancer : Clinical course and characteristics
}

Ji-Geun Yoo, Yoon-Jin Choi, Jong-Sup Park, Keun-Ho Lee, Soo-Young Hur Department of Obstetrics and Gynecology, Seoul St. Mary's Hospitak,

The Catholic University of Korea

\section{Introduction}

For advanced gynecologic cancer patients, Chemotherapy has provided survival benefits to cancer patients, but at the same time has a risk of bone marrow suppression and possibility of combined infection, and occasionally cause lethal septic shock, which is associated with a mortality rate of $40 \%$. Still, there is only a few reports about chemotherapy-related neutropenic septic shock in gynecologic malignancies. The purpose of this study is to assess the risk and determine clinical characteristics and course of septic shock in gynecologic cancer patients.

\section{Methods}

In this single-center retrospective study we reviewed medical records of gynecologic cancer patients who had treated with chemotherapeutic agents between Jan. 2009 and Dec. 2017. Neutropenic septic shock is defined as neutropenia $<1 \mathrm{~g} / \mathrm{L}$ with septic shock that requires inotropics to maintain normal blood pressure. We divided the events into two groups according to survival. Statistical analysis was performed for the clinical characteristics by Mann-Whitney U-test.

\section{Results}

Total 1363 patients received at least 1 cycle of chemotherapy for gynecologic malignancies. Among them, 22(1.6\%) patients experienced 25 events of neutropenic septic shock. Six events of death have occurred, and the mortality ratio was $24 \%$.

Table 1. Clinical characteristics of the events.

\begin{tabular}{|c|c|c|c|c|}
\hline Characteristics & Total & Events of survive & Events of death & $P$ value \\
\hline Events, $\mathrm{n}$ & 25 & $19(76.0 \%)$ & $6(24.0 \%)$ & \\
\hline Median age, yrs & 59 [29-78] & 57 [29-66] & $62[55-78]$ & .085 \\
\hline Mean BMI, kg/m² & $22.9[14.7-28.4]$ & $22.9[14.7-28.4]$ & $22.7[18.2-27.5]$ & .874 \\
\hline \multicolumn{5}{|l|}{ Type of cancer } \\
\hline Ovarian & $13(52.0 \%)$ & $9(47.4 \%)$ & $4(66.7 \%)$ & \\
\hline Endometrial & $6(24.0 \%)$ & $4(21.1 \%)$ & $2(33.3 \%)$ & \\
\hline Peritoneal & $3(12.0 \%)$ & $3(15.8 \%)$ & & \\
\hline Cervical & $2(8.0 \%)$ & $2(10.5 \%)$ & & \\
\hline Uterine leiomyosarcoma & $1(4.0 \%)$ & $1(5.3 \%)$ & & \\
\hline \multicolumn{5}{|l|}{ FIGO Stage, n(\%) } \\
\hline 1 & $1(4.0 \%)$ & $1(5.3 \%)$ & 0 & \\
\hline II & $4(16.0 \%)$ & $3(15.8 \%)$ & $1(16.7 \%)$ & \\
\hline III & $11(44.0 \%)$ & $7(36.8 \%)$ & $4(66.7 \%)$ & \\
\hline IV & $9(36.0 \%)$ & $8(42.1 \%)$ & $1(16.7 \%)$ & \\
\hline \multicolumn{5}{|l|}{ Recurrence status } \\
\hline Primary treatment, n(\%) & $8(32.0 \%)$ & $6(31.6 \%)$ & $2(33.3 \%)$ & \\
\hline Recurrent disease, n(\%) & $17(68.0 \%)$ & $13(68.4 \%)$ & $4(66.7 \%)$ & \\
\hline Duration from the initial chemotherapy(mo.) & 23 [1-131] & 18 [1-85] & 28 [1-131] & .799 \\
\hline Days from the latest chemotherapy(d.) & 8 [3-14] & 8 [3-14] & 8 [4-14] & .923 \\
\hline Peak body temperature* ${ }^{\star}(' \mathrm{C})$ & $38.8[35.6-40.4]$ & $39[37.3-40.4]$ & $38.3[35.6-40.4]$ & .251 \\
\hline Mean absolute neutrophil count*, mg/L & $150[0-780]$ & $122.6[0-550]$ & $236.7[0-780]$ & .305 \\
\hline Mean CRP at diagnosis, $\mathrm{mg} / \mathrm{dL}$ & $17.8[1.3-30.4]$ & $17.4[1.28-29.1]$ & $18.9[9.49-30.4]$ & .703 \\
\hline Median duration of neutropenia, days & $3[1-7]$ & $4[1-7]$ & $3[1-6]$ & \\
\hline Mean duration of inotropics, $\mathrm{Hr}$ & $50.6[6-191.9]$ & $49.3[6-140.8]$ & $54.8[11-191.9]$ & .899 \\
\hline
\end{tabular}
${ }^{*}$ The lowest or highest value during event
There was no significant difference between events of death group and survive group according to age, BMI, FIGO stage, mean CRP at the time of diagnosis of septic shock, the lowest neutrophil count during the event, and peak body temperature. Six events occurred during the primary treatment course. We experienced two events of septic shock who received chemotherapeutic agents only once. According to the chemotherapeutic regimen, carboplatin-paclitaxel combination chemotherapy accounted for the largest proportion(24\%).

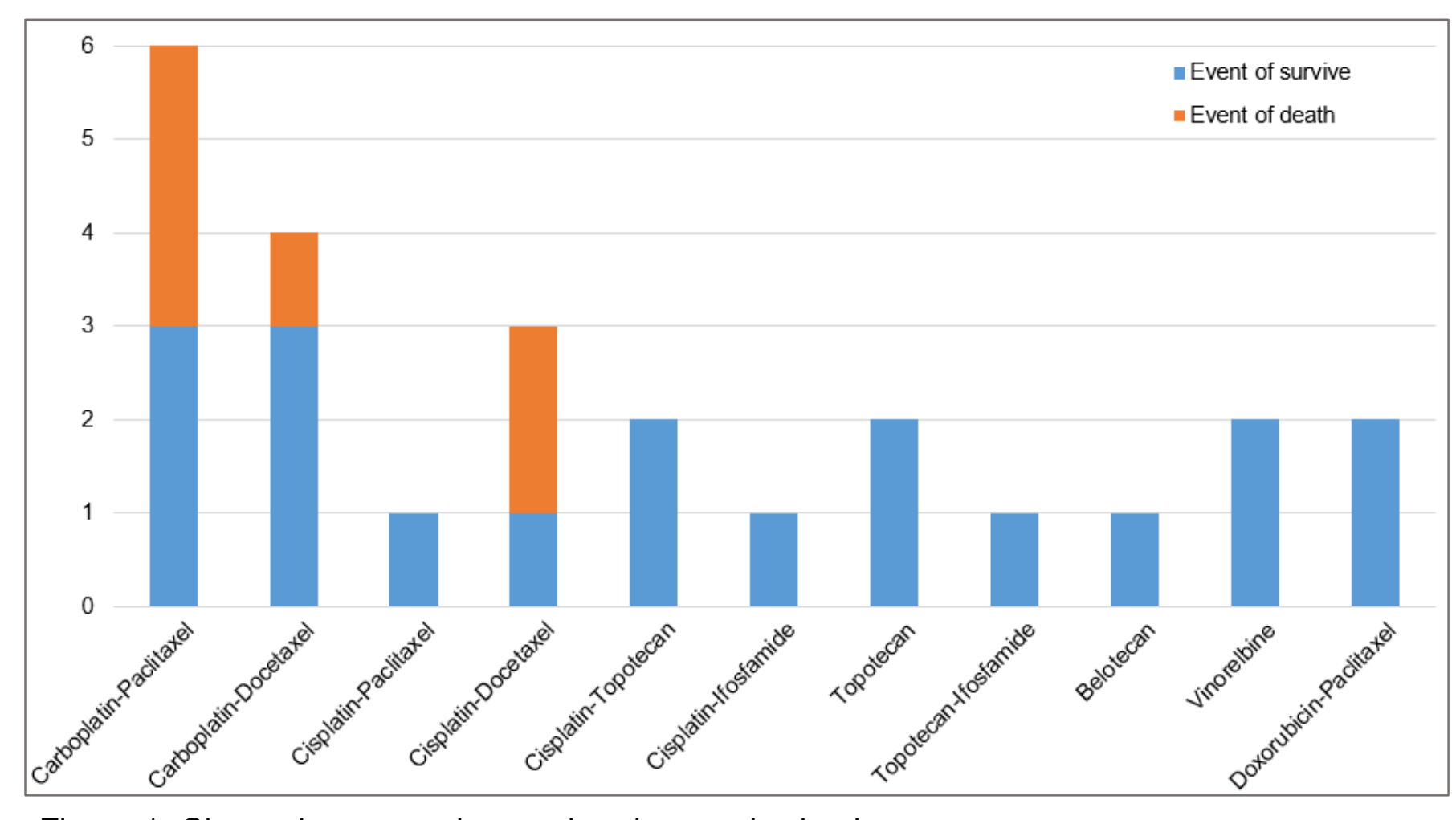

Figure 1. Chemotherapy regimen related to septic shock.

Two-thirds of events had identified bacteria on blood culture. Most common pathogen isolated was $\mathrm{E}$. coli( $7 / 16,43.7 \%)$.

In the event of survivor group, mean recovery time was 2 days of initial treatment. Meanwhile, for events of death group, the average time from initial treatment of septic shock to death was 54.8 hours.

Table 2. Identified pathogen on blood culture

\begin{tabular}{llcc}
\hline & Total $(\mathrm{n}=25)$ & Events of survive $(\mathrm{n}=19)$ & Events of death $(\mathrm{n}=6)$ \\
\hline E. Coli & $7(28.0 \%)$ & $5(26.3 \%)$ & $2(33.3 \%)$ \\
ESBL(-) & $5(20.0 \%)$ & $3(15.8 \%)$ & $2(33.3 \%)$ \\
ESBL(+) & $2(8.0 \%)$ & $2(10.5 \%)$ & 0 \\
Enterobacter Cloacae & $2(8.0 \%)$ & $2(10.5 \%)$ & 0 \\
Klebsiella Pneumoniae & $3(12.0 \%)$ & $2(10.5 \%)$ & $1(16.7 \%)$ \\
Pseudomonas Aeruginosa & $1(4.0 \%)$ & 0 & $1(16.7 \%)$ \\
Enterococcus Faecalis & $1(4.0 \%)$ & 0 & $1(16.7 \%)$ \\
Others & $2(8.0 \%)$ & $2(10.5 \%)$ & 0 \\
Not identified & $9(36.0 \%)$ & $8(42.1 \%)$ & $1(16.7 \%)$ \\
\hline
\end{tabular}

\section{Conclusion}

Through this study, we confirmed the incidence and mortality, and clinical course of neutropenic septic shock in gynecologic malignancy. We hope that this study will provide clinicians with information about chemotherapy related septic shock and help patients recover from this lethal disease. 